\title{
EXTENSIONS OF MINIMAL FLOWS ON MANIFOLDS
}

\author{
WILLIAM PERRIZO ${ }^{1}$
}

\begin{abstract}
Group extensions of vector field flows for which the orbit closure map forms a fiber bundle are constructed for the case of minimal flows on compact manifolds and compact Lie groups. Conditions for which minimal nontoral extensions exist are studied.
\end{abstract}

The existence question for minimal sets and minimal extensions of minimal sets has been studied extensively in topological dynamics. In [5], Ellis considers the group extension question for minimal homeomorphisms on compact metric spaces. For real flows on compact manifolds it is well known that the torus admits a minimal flow but the Klein bottle does not. In fact, Markley [7] shows that recurrent orbits on the Klein bottle are circles or points. It is also known that a minimal vector field flow admits a minimal extension through any torus [1, p. 58]. The purpose of this paper is to prove that a minimal vector field flow admits a minimal extension beyond a toral extension. It will follow that any Lie group admits a vector field flow which is minimal on the product of two distinct maximal tori and that any minimal vector field flow admits a minimal compact group extension in which the group is a semidirect product with a torus.

It is also shown that a minimal vector field flow can always be extended so that the minimal sets form a partition into imbedded submanifolds and the projection onto the orbit closure spaces forms a fiber bundle.

0. Preliminaries. Definitions and proofs omitted here can be found in [1], [3] and [4]. All manifolds, maps, and transformation groups will be assumed $C^{\infty}$ unless otherwise specified.

If $(M, G)$ is right transformation group, the set $\{n \cdot g \mid n \in N \subseteq M, g \in G\}$ will be denoted by $N \cdot G$ and the space $\{n \cdot G \mid n \in N \subseteq M\}$ with the quotient topology will be denoted by $N / G$. If $G$ is the real line and $X$ is the vector field generating $(M, G)$, we denote $(M, G)$ by $(M, X), N \cdot G$ by $N \cdot X$ and $N / G$ by $N / X$. The symbols $G \cdot N$ and $G \backslash N$ will be used in case $G$ acts on the left. If $(G, P, M, \pi)$ is a principal $G$-bundle with base $M$, bundle space $P$ and projection $\pi ;\left(H, P, H \backslash P, \pi_{1}\right)$ will denote the induced principal $H$-bundle in which $\pi_{1}: P \rightarrow H \backslash P$ takes $p$ to $H \cdot p$. $\left(H \backslash G, H \backslash P, M, \pi_{2}\right)$ will denote the induced fiber bundle with standard fiber $H \backslash G$ and projection $\pi_{2}$ : $H \backslash P \rightarrow M$ which takes $H \cdot p$ to $\pi(p)$. Note that $\pi=\pi_{2} \circ \pi_{1}$. For any map $f$,

Received by the editors September 28, 1975.

AMS (MOS) subject classifications (1970). Primary 54H20, 34H35; Secondary 58F99.

Key words and phrases. Minimal, transitive, almost periodic, vector field flow, $G$-lift, fiber bundle, Lie group.

${ }^{1}$ Research supported in part by NSF Institutional Grant for Science, GU 6315, North Dakota State University. 
$f_{*}$ will denote the space derivative of $f$. A vector field $Y$ on $P$ will be called a lift of a vector field $X$ on $M$ if $\pi_{*} Y=X$ and a $G$-lift if it is a lift which is invariant under the action of $G$ on $P$. Let $(M, G)$ be a transformation group. A set $A \subseteq G$ is syndetic if there is a compact set $K \subseteq G$ such that $A \cdot K=G$. A point $x$ is $G$-almost periodic if the set $\{g \in G \mid x \cdot g \in U\}$ is syndetic for each open set $U$ containing $x .(M, G)$ is $G$-weakly almost periodic ( $G$-uniformly almost periodic) if for each index $\alpha, \exists K$ compact $\ni:\{g \in G \mid x \cdot g \in x \alpha\} \cdot K$ $=G$ for each $x \in M\left(\cap_{x \in M}\{g \mid x g \in x \alpha\} K=G\right)$. A set $N \subseteq M$ is $G$ transitive (G-minimal) if $\overline{x \cdot G}=N$ for some $x \in N$ (for all $x \in N$ ). Fiber bundles will be denoted $(F, P, M, \pi)$ where $F$ is the fiber, $P$ the bundle manifold, $M$ the base manifold and $\pi$ the projection.

\section{The existence of $G$-lifts in which the orbit closure partition forms a fiber bundle.}

1.1. Theorem. Let $P$ be compact, $(G, P, M, \pi)$ a principal bundle, and $X$ a minimal vector field on $M$. Given $p \in P$, there exists a $G$-lift $Y$ such that

(i) $\{\overline{(g \cdot p) \cdot Y} \mid g \in G\}$ partitions $P$ into imbedded submanifolds.

(ii) The sets $G_{q}=\{g \in G \mid g \cdot q \in \overline{q \cdot Y}\}$ are closed subgroups of $G$.

(iii) $\left(G_{p}, \overline{p \cdot Y}, M, \pi\right)$ is a reduction of the bundle $(G, P, M, \pi)$.

(iv) The map $\phi: P \rightarrow G / G_{p}$, taking $q$ to $[g]=g \cdot G_{p}$ iff $q \in \overline{(g \cdot p) \cdot Y}$, forms a fiber bundle $\left(\overline{p \cdot Y}, P, G / G_{p}, \phi\right)$.

By the structure theorem for compact group extensions of minimal transformation groups $\left[1\right.$, p. 46], the orbit closures partition $P$ into minimal sets, $G_{q}$ is a closed subgroup, and $\phi$ is continuous.

The space $p \cdot Y$ is a $G_{p}$-invariant set and $G_{p} \backslash p \cdot Y=M$. By Gleason's cross section theorem [2], there exist continuous local cross sections of $\pi$ : $\overline{p \cdot Y} \rightarrow M$.

1.2. Lemma. Let $Y$ be $a$ G-lift of $X$ and $G_{p}=\{g \in G \mid g \cdot p \in \overline{p \cdot Y}\}$. The map $\sigma(x)=\pi_{2}^{-1}(x) \cap \pi_{1}(\overline{p \cdot Y})$ of $M$ into $G_{p} \backslash P$ is a continuous cross section.

Proof OF 1.2. For each $q=\lim _{\alpha} Y_{t_{\alpha}}(p)$ from

$$
\begin{aligned}
\pi^{-1}(x) & \cap \overline{p \cdot Y}=\pi^{-1}(x) \cap \overline{q \cdot Y}=G_{q} \cdot q, \\
g \in G_{q} & \Leftrightarrow g \cdot q=g \cdot \lim _{\alpha} Y_{t_{\alpha}}(p) \in \overline{q \cdot Y}=\overline{p \cdot Y} \\
& \Leftrightarrow \lim Y_{t_{\alpha}}(g \cdot p) \in \overline{p \cdot Y} \\
& \Leftrightarrow g \cdot p \in \overline{p \cdot Y} \Leftrightarrow g \in G_{p} .
\end{aligned}
$$

Thus, $G_{q}=G_{p}, G_{q} \cdot q=G_{p} \cdot q$, and

$$
\begin{aligned}
\pi_{1}(q) & =\pi_{1}\left(G_{p} \cdot q\right)=\pi_{1}\left(\pi^{-1}(x) \cap \overline{p \cdot Y}\right)=\pi_{1} \circ \pi^{-1}(x) \cap \pi_{1}(\overline{p \cdot Y}) \\
& =\pi_{2}^{-1}(x) \cap \pi_{1}(\overline{p \cdot Y})=\sigma(x) .
\end{aligned}
$$

Thus, $\sigma$ is well defined. Locally, $\sigma$ is the composition of a cross section of $\pi$ which takes its values in $\overline{p \cdot Y}$ with the map $\pi_{1}$. Thus, $\sigma$ is continuous.

1.3. Lemma. Let $\left(G^{n}, P^{n}, M, \pi^{n}\right)$ be a reduction of $(G, P, M, \pi)$ and let $Y^{n}$ be a $G$-lift of $X$ such that $p \in P^{n} \subseteq P, \pi^{n}=\left.\pi\right|_{P^{n}}$, and $P^{n}$ is $Y^{n}$-invariant. If 
$G^{n+1}=\left\{g \in G \mid g \cdot p \in \overline{p \cdot Y^{n}}\right\}$, then there is a reduction $\left(G^{n+1}, P^{n+1}, M\right.$, $\left.\pi^{n+1}\right)$ of $(G, P, M, \pi)$ and a $G$-lift $Y^{n+1}$ of $X$ such that $p \in P^{n+1} \subseteq P$, $\pi^{n+1}=\left.\pi\right|_{P^{n+1}}$ and $P^{n+1}$ is $Y^{n+1}$-invariant.

Proof of 1.3. Let $\sigma^{n}$ be the continuous cross section into $G^{n+1} \backslash P$ as in 1.2. Let $\delta^{n}$ be a $C^{\infty}$ approximation of $\sigma^{n}\left[3\right.$, p. 25]. Define $P^{n+1}$ $=\left(\pi^{n}\right)_{1}^{-1}\left(\delta^{n}(M)\right)$ and define $Y^{n+1}$ to be any $G^{n+1}$-lift of $\delta_{*}^{n} X$. The result follows.

By iterating 1.3 , a sequence of reductions $(G, P, M, \pi),\left(G^{1}, P^{1}, M\right.$, $\left.\pi^{1}\right), \ldots,\left(G^{n}, P^{n}, M, \pi^{n}\right), \ldots$ is obtained such that $G \supseteq G^{1} \supseteq \cdots$ $\supseteq G^{n} \ldots$

1.4. Lemma. There is an $n$ such that $G^{n}=G^{n+1}$.

Proof of 1.4. If $G^{n} \neq G^{n+1}$ and $\operatorname{dim}\left(G^{n}\right)=\operatorname{dim}\left(G^{n+1}\right)$, then the finite set $G^{n+1} / C$ is $\subseteq G^{n} / C$, where $C$ is the identity component of both $G^{n+1}$ and $G^{n}$. Thus, $G^{n+1}$ and $G^{n}$ can differ for at most a finite number of stages.

If $G^{n+1}=G^{n}, Y^{n}$ is minimal on $P^{n}$ and on $g \cdot P^{n}$ for every $g \in G$ as well. Let $\delta^{n}=\sigma^{n}$, then $Y^{n}$ is a $G$-lift of $X$ satisfying (i), (ii), and (iii) of 1.1. The following lemma completes the proof of 1.1 .

1.5. Lemma. If $Y$ is a G-lift of $X$ satisfying (i), (ii), and (iii) of 1.1 and $\phi$ : $P \rightarrow G / G_{p}$ is the orbit closure map of (iv), then $\left(\overline{p \cdot Y}, P, G / G_{p}, \phi\right)$ forms a fiber bundle.

Proof of 1.5. Let $\Gamma: W \times G_{p} \rightarrow \operatorname{pr}^{-1}(W)$ and $\psi: G_{p} \times U \rightarrow \psi^{-1}(U)$ be local trivializing diffeomorphisms of the bundles $\left(G_{p}, G, G / G_{p}\right.$, pr) and $\left(G_{p}\right.$, $\overline{p \cdot Y}, M, \pi)$ at the points $[g]$ and $x$ respectively. Define $\theta: W \times \overline{p \cdot Y}$ $\rightarrow \phi^{-1}(W)$ by $\theta([h], q)=\Gamma([h], e) \cdot q$. Since $\phi \circ \theta=$ projection onto the first factor, $\theta([h], q)=\theta\left(\left[h^{\prime}\right], q^{\prime}\right)$ implies $[h]=\left[h^{\prime}\right]$; which implies $\Gamma([h], e)$ $=\Gamma\left(\left[h^{\prime}\right], e\right)$; which implies $q=q^{\prime}$. Thus, $\theta$ is one-to-one. An element $q \in$ $\phi^{-1}(W)$ is in $\overline{(h \cdot p) \cdot Y}$ for some $h \in G$ such that $[h] \in W$. That is, $q=h q^{\prime}$ for some $q^{\prime}$ in $\overline{p \cdot Y}$ and $h=\Gamma([h], b)$. Thus,

$$
\theta\left([h], b \cdot q^{\prime}\right)=\Gamma([h], e) \cdot\left(b \cdot q^{\prime}\right)=\Gamma([h], b) q^{\prime}=h q^{\prime}=q .
$$

Thus, $\theta$ is onto. On $W \times \pi^{-1}(U), \theta$ is the composition of the following diffeomorphisms:

$$
\begin{gathered}
\mathrm{id} \times \psi^{-1}: G / G_{p} \times \overline{p \cdot Y} \rightarrow G / G_{p} \times G_{p} \times U, \\
\Gamma \times \mathrm{id}: G / G_{p} \times G_{p} \times U \rightarrow G \times U, \quad \psi: G \times U \rightarrow P, \\
([h], q) \rightarrow([h], b, \pi(q)) \rightarrow(\Gamma([h], b), \pi(q)) \rightarrow \psi(\Gamma([h], b), \pi(q)) \\
=\Gamma([h], e) \cdot q=\theta([h], q) .
\end{gathered}
$$

Thus, $\theta$ is a local trivializing diffeomorphism for $\phi$ at $[g]$.

1.6. Theorem. Let $Y$ be a $G$-lift of $X$ as in 1.1. If $Z$ is a vector field on $P$ such that $[Y, Z]=0$, then $\phi_{*} Z$ exists. If $Z_{1}$ is a vector field on $G / G_{p}$, there exists $Z$ on $P$ such that $\phi_{*} Z=Z_{1}$. Furthermore, if $Z_{1}$ is $G$-invariant, $Z$ can be constructed G-invariant.

Proof OF 1.6. If $[Y, Z]=0$, 


$$
\phi_{*}\left(Z\left(Y_{s}(q)\right)\right)=\left.\frac{d}{d t}\left(\phi \circ Z_{t} \circ Y_{s}(q)\right)\right|_{t=0}=\left.\frac{d}{d t}\left(\phi \circ Z_{t}(q)\right)\right|_{t=0}=\phi_{*}(Z(q)) .
$$

Since $\phi_{*} Z$ is a continuous function, it is constant on $\overline{q \cdot Y}=\phi^{-1}(\phi(q))$. Thus, $\phi_{*} Z$ defines a vector field on $G / G_{p}$.

Let $\left\{\alpha_{i}: W_{i} \times \overline{p \cdot Y} \rightarrow \phi^{-1}(W)\right\}$ be a cover of trivializing diffeomorphisms and let $\left\{\delta_{i}\right\}$ be a partition of unity subordinate to $\left\{W_{i}\right\}$. Given $Z_{1}$ on $G / G_{p}$, define $Z_{i}$ on $\phi^{-1}\left(W_{i}\right)$ as $\alpha_{i}\left(Z_{1}, 0\right)$ and define $Z$ as $\Sigma\left(\delta_{i} \circ \phi\right) \cdot Z_{i}$. Clearly, [ $Y$, $Z]=0$ and $\phi_{*} Z=Z_{1}$.

\section{Extending almost periodicity and transitivity.}

2.1. TheOREM. Let $Y$ and $Z$ be vector fields on the compact manifold $P$. If $p \in P$ is $Z$-almost periodic, $(P, Y)$ weakly almost periodic and $[Y, Z]=0$, then $\{a \mid \overline{(p \cdot Y)} \cdot Z$ is $(Y+a Z)$-transitive $\}$ is a comeager set of real numbers.

2.2. LEMMA. $\overline{(\overline{p \cdot Y}) \cdot Z}=\overline{(p \cdot Y) \cdot Z}$ and if $[Y, Z]=0$, this set coincides with $\overline{\overline{(p \cdot Z) \cdot Y}}=\overline{(p \cdot Z) \cdot Y}$.

PROOF OF 2.2. Since $q \in(\overline{p \cdot Y}) \cdot Z$ iff $q=Z_{t}\left(\lim _{\alpha} Y_{s_{\alpha}}(p)\right)$ $=\lim _{\alpha} Z_{t} \circ Y_{s_{\alpha}}(p)$ and $q \in \overline{(p \cdot Y) \cdot Z}$ iff $q=\lim _{\beta} Z_{t_{\beta}} \circ Y_{s_{\beta}}(p)$, $\overline{(p \cdot Y)} \cdot Z \subseteq \overline{(p \cdot Y) \cdot Z}$. Thus, $\overline{(p \cdot Y) \cdot Z} \subseteq \overline{(p \cdot Y) \cdot Z}$. Since $\overline{(p \cdot Y) \cdot Z}$ $\supseteq(p \cdot Y) \cdot Z, \overline{(p \cdot Y) \cdot Z} \supseteq \overline{(p \cdot Y) \cdot Z}$ and the lemma follows.

Proof of 2.1. Let $\left\{U_{i} \mid i \in I\right\}$ be a countable base for the topology of $P$. If $V_{i}$ is an open set such that $\bar{V}_{i} \subseteq U_{i}$ and $V_{i} \cap \overline{(p \cdot Z) \cdot Y} \neq \varnothing$, then $V_{i} \cap(\overline{p \cdot Z}) \cdot Y \neq 0$ and $V_{i} \cdot Y \cap p Z \neq \varnothing$. Let $E\left(U_{i}\right)=\{a \mid p \cdot(a Y+Z) \cap$ $\left.U_{i} \neq \varnothing\right\}$. Each $E\left(U_{i}\right)$ is an open set since small perturbations of vector fields produce small changes in the flow transformations. Since $p$ is $Z$-almost periodic, there is a sequence $t_{n} \rightarrow+\infty$ such that $Z_{t_{n}}(p) \in V_{i} \cdot Y$. Thus, $(a Y+Z)_{t_{n}}(p)=Y_{a \cdot t_{n}} \circ Z_{t_{n}}(p) \in V_{i} \cdot Y$. By passing to a subsequence,

$$
(a Y+Z)_{t_{n}}(p) \rightarrow p_{1} \in \overline{V_{i} \cdot Y} .
$$

Since $(P, Y)$ is weakly almost periodic, $\overline{V_{i} \cdot Y}=\cup_{q \in \bar{V}_{i}} \overline{q \cdot Y}[6$, p. 34]. Thus, there exists a $q \in \bar{V}_{i}$ such that $p_{1} \in \overline{q \cdot Y}$. Since $q$ is $Y$-almost periodic, $q \in \overline{p_{1} \cdot Y}$ and $\exists s$ such that $Y_{s}\left(p_{1}\right) \in U_{i}$. Passing to a subsequence once again, $(a Y+Z)_{t_{n}}(p) \in Y_{-s}\left(U_{i}\right)$.

Given $\varepsilon>0$, choose $|b|<\varepsilon$ and $t$ from $\left\{t_{n}\right\}$ such that $s=b t$. It follows that

$$
((a+b) Y+Z)_{t}(p)=Y_{b t}\left((a Y+Z)_{t}(p)\right) \in Y_{s}\left(Y_{-s}\left(U_{i}\right)\right)=U_{i} .
$$

Thus, $\{a \mid \overline{(\overline{p \cdot Y}) \cdot Z}$ is $(a Y+Z)$-transitive $\}$ is comeager. Since $Y+a Z$ $=a\left(a^{-1} Y+Z\right)$, the result follows.

2.3. TheOREM. Let $Y$ and $Z$ be vector fields on $P$ such that $[Y, Z]=0$. If $p$ is $Y$-almost periodic, $(\overline{p \cdot Z}, Z)$ is uniformly almost periodic and $(P, Z)$ is weakly almost periodic, then $p$ is $(Y+a Z)$-almost periodic.

2.4. Lemma. If $p$ is $Y$-almost periodic, each point of $\overline{p \cdot Y}$ is Z-almost periodic, then $\overline{(q \cdot Y) \cdot Z}=\overline{(p \cdot Y) \cdot Z}$ for every $q \in \overline{(p \cdot Y) \cdot Z}$ ( $p$ is an almost periodic point of $\left(P, \mathbf{R}^{2}\right)$ given by $\left.p \cdot(s, t)=Z_{t} \circ Y_{s}(p)\right)$. 


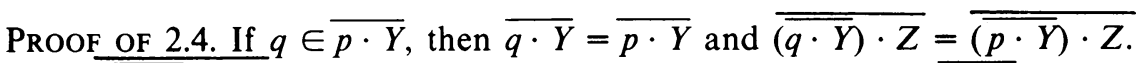
If $q \in \overline{(\overline{p \cdot Y}) \cdot Z}$, then $q=\lim _{\alpha} Z_{t_{\alpha}}\left(q^{\prime}\right)$ for some $q^{\prime}$ in $\overline{p \cdot Y}$. Since $\overline{q \cdot Z}=\overline{q^{\prime} \cdot Z}, \overline{(\overline{q \cdot Z}) \cdot Y}=\overline{\left(q^{\prime} \cdot Z\right) \cdot Y}=\overline{(\overline{p \cdot Z}) \cdot Y}$. Thus, $\overline{(q \cdot Z) \cdot Y}=\overline{(p \cdot Z) \cdot Y}$.

Proof OF 2.3. Let $q$ be a $(Y+a Z)$-almost periodic point of $\overline{p \cdot(Y+a Z)}$. By $2.4, p \in \overline{\overline{(p \cdot(Y+a Z))} \cdot Z}=\overline{q \cdot(Y+a Z) \cdot Z}$. Since $(P, Z)$ is weakly almost periodic, $\overline{q \cdot(Y+a Z) \cdot Z}=\bigcup\{\overline{x \cdot Z} \mid x$ in $\overline{q \cdot(Y+a Z)}\}$. Thus, there is $x \in \overline{q \cdot(Y+a Z)}$ such that $p \in \overline{x \cdot Z}$. Since $Z$ is uniformly almost periodic on $\overline{p \cdot Z}=\overline{x \cdot Z}$, there is an element, $f$, of the enveloping semigroup of $(P, Z)$ which is continuous at $x$ and takes $x$ to $p[1$, pp. 17, 25]. Suppose $f$ $=\lim _{\alpha} Z_{s_{\alpha}}$; then

$$
\begin{aligned}
f(Y+a Z)_{t}(x) & =\lim Z_{s_{\alpha}} \circ(Y+a Z)_{t}(x) \\
& =(Y+a Z)_{t}\left(\lim _{\alpha} Z_{s_{\alpha}}(x)\right)=(Y+a Z)_{t}(f(x)) .
\end{aligned}
$$

Thus, $f$ takes the $(Y+a Z)$-almost periodic point, $x$, to a $(Y+a Z)$-almost periodic point, namely $p[1$, p. 11].

\section{Applications.}

3.1. Theorem. Let $(G, P, M, \pi)$ be a principal bundle with $P$ compact, $X$ a minimal vector field on $M$ and $Y$ a $G$-lift of $X$. If $Y$ induces a fiber bundle $\left(\overline{p \cdot Y}, P, G / G_{p}, \phi\right)$ as in 1.1 and $Z_{1}$ is a $G$-invariant vector field on $G / G_{p}$, then there is a G-lift $Y_{1}$ of $X$ such that for each $[h]$ in $G / G_{p}, \phi^{-1}\left(\overline{\left([h] \cdot Z_{1}\right)}\right)$ is a $Y_{1}$-minimal set.

Proof of 3.1. Let $Z$ be a $G$-invariant vector field on $P$ such that $\phi_{*} Z=Z_{1}$ and $[Y, Z]=0$ as in 1.6. $Z$ and $Y+a Z$ are pointwise almost periodic since both are $G$-lifts of pointwise almost periodic transformation groups. Since $\phi^{-1}\left(\overline{\left([h] \cdot Z_{1}\right)}\right)=\overline{(q \cdot Y) \cdot Z}$ for each $q \in \phi^{-1}([h]),\left\{a \mid \phi^{-1}\left(\overline{\left([h] \cdot Z_{1}\right)}\right)\right.$ is $(Y+a Z)$-minimal $\}$ is comeager, by 2.1 .

3.2. LeMMA. Let $(G, P, M, \pi)$ be a principal bundle with compact bundle space, $P$. Let $A$ be a Lie algebra element of $G$. $A$ induces a vector field $A^{*}$ on $P$ with flow $A_{t}^{*}(p)=\exp (A t) \cdot p$ which is weakly almost periodic and uniformly almost periodic on each of its orbit closures. Furthermore, each $A^{*}$ commutes with each $G$-lift, $Y$.

Proof of 3.2. If $T=\overline{\{\exp (A t) \mid t \text { real }\}}$ in $G$, then $T \cdot p=\overline{p \cdot A^{*}}$ for each $p \in P$. Let $C$ be closed in $P$ and $\left(T, P, T \backslash P, \pi_{1}\right)$ be the principal bundle induced by action of $T$. Since $\pi_{1}$ is a continuous closed map, $\pi_{1}(C)$ and $\pi^{-1}(\pi C)=T \cdot C=C \cdot A^{*}$ are closed sets. Thus, $\left(P, A^{*}\right)$ is weakly almost periodic [4, p. 34]. Since $A$ induces an equicontinuous rotational flow on the torus $T$ and since $*:(T, A) \rightarrow\left(T \cdot p, A^{*}\right)$ is an isomorphism, $A^{*}$ is uniformly almost periodic on its orbit closure $T \cdot p=\overline{p \cdot A^{*}}$ for each $p$ in $P$.

3.3. Corollary. Let $(G, P, M, \pi)$ be a principal bundle with compact bundle space, $X$ a minimal vector field on $M$ and $Y$ a $G$-lift of $X$. Given any Lie algebra element $A$ of $G$, there exists a lift $Y_{1}$ of $X$ such that each set $\overline{(q \cdot Y) \cdot A^{*}}$ is a $Y_{1}$-minimal set. 
Proof of 3.3. The corollary follows immediately from 2.1, 2.3, and 3.2.

3.4. THEOREM. Let $G$ be a compact Lie group such that the identity component is abelian, let $P$ be compact and connected and let $(G, P, M, \pi)$ be a principal bundle. If there exists a ninimal vector field $X$ on $M$, there exists a minimal vector field $Y$ on $P$ which is a lift of $X$.

Proof of 3.4. Let $Y_{1}$ be a $G$-lift of $X$ which induces a fiber bundle $(\overline{p \cdot Y}$, $\left.P, G / G_{p}, \phi\right)$ as in 1.1. Since $P$ is connected, $\phi(P)=G / G_{p}$ is connected. The canonical projection, pr: $G \rightarrow G / G_{p}$ is a continuous, open and closed map. If $G_{0}$ is the identity component of $G, \operatorname{pr}\left(G_{0}\right)$ is open and closed in $G / G_{p}$ and is therefore equal to $G / G_{p}$. Thus, $G_{0} \cdot G_{p}=G$. Let $A$ be a Lie algebra element such that $\overline{e \cdot A}=G_{0}$; then there is a vector field $Y$ on $P$ such that $\overline{\left(p \cdot A^{*}\right) \cdot Y_{1}}$ is $Y$-minimal, by 3.3 . Since

$$
\overline{\left(p \cdot A^{*}\right) \cdot Y}=\overline{\left(\overline{p \cdot A^{*}}\right) \cdot Y}=\overline{\left(G_{0} \cdot p\right) \cdot Y}=\phi^{-1}\left(\phi\left(G_{0} \cdot p\right)\right)
$$

and $\phi\left(G_{0} \cdot p\right)=G / G_{p},\left(p \cdot A^{*}\right) \cdot Y=P$.

3.5. Corollary. Let $G$ be a semidirect product of a torus and a finite group and let $(G, P, M, \pi)$ be a principal bundle such that $P$ is compact and connected. If there exists a minimal vector field $X$ on $M$, there exists a minimal lift of $X$ on $P$.

Proof OF 3.5. This result follows from 3.4 since the identity component of $G$ is abelian.

3.6. Corollary. Let $T$ and $S$ be distinct maximal tori in a Lie group $G$. There exists a vector field $Y$ on $G$ such that each $g \cdot T \cdot S$ is a $Y$-minimal set.

Proof of 3.6. Let $M=\{e\}$ and $(G, G, M, \pi)$ be the trivial principal bundle on $M$. Let $A$ and $B$ be Lie algebra elements of $G$ such that $A$ is minimal on $S$ and $B$ is minimal on $T$. Define $Y_{1}$ on $G$ to be $B$. Clearly, $Y_{1}$ is a $G$-lift of 0 since $L_{g_{*}} \cdot Y_{1}=Y_{1}$ by definition. Thus, by 3.3 , the corollary follows. (Note $A^{*}$ is right invariant so $\left[B, A^{*}\right]=0$.)

\section{REFERENCES}

1. R. Ellis, Lectures on topological dynamics, Benjamin, New York, 1969. MR 42 \#2463.

2. A. M. Gleason, Spaces with a compact Lie group of transformations, Proc. Amer. Math. Soc. 1 (1950), 35-43. MR 11, 497.

3. N. E. Steenrod, The topology of fibre bundles, Princeton Math. Ser., vol. 14, Princeton Univ. Press, Princeton, N. J., 1951. MR 12, 522.

4. W. Gottschalk and G. Hedlund, Topological dynamics, Amer. Math. Soc. Colloq. Publ., vol. 36, Amer. Math. Soc., Providence, R. I., 1955. MR 17, 650.

5. R. Ellis, The construction of minimal discrete flows, Amer. J. Math. 87 (1965), 564-574. MR 32 \# 3053.

6. N. G. Markley, Lifting dynamical properties, Math. Systems Theory, 5 (1971), 229-305. MR $46 \# 4514$.

7. The Poincare-Bendixson theorem for the Klein bottle, Trans. Amer. Math. Soc. 135 (1969), 159-165. MR 38 \#2759.

Department of Mathematics, North Dakota State University, Fargo, North Dakota 58102 